\title{
Immune response to SARS-CoV-2 vaccination among renal replacement therapy patients with CKD: a single-center study
}

\author{
Masatoshi Matsunami ${ }^{1}$ (D) Tomo Suzuki $^{1} \cdot$ Toshiki Terao $^{2} \cdot$ Hiroshi Kuji $^{3} \cdot$ Kosei Matsue $^{2}$
}

Received: 3 October 2021 / Accepted: 1 November 2021 / Published online: 8 November 2021

(c) Japanese Society of Nephrology 2021

Keywords COVID-19 $\cdot$ SARS-CoV-2 $\cdot$ Vaccine $\cdot$ Hemodialysis $\cdot$ Peritoneal dialysis $\cdot$ Kidney transplantation

\section{To the Editor}

Generally, immune response to vaccination is less robust in chronic kidney disease (CKD) patients than in healthy patients. Therefore, prophylactic vaccination against severe acute respiratory syndrome coronavirus 2 (SARS-CoV-2) is an important component particularly for CKD patients, receiving renal replacement therapy (RRT). Until now, studies investigating the immune response to SARS-CoV-2 for these patients are limited [1-5]. We evaluated the immune response to SARS-CoV-2 vaccination among dialysis patients and kidney transplant recipients, and compared them with a control group.

We performed a retrospective single-center study, evaluating antibody responses among 78 hemodialysis (HD) patients, 27 peritoneal dialysis (PD) patients, and 21 kidney transplant (KTx) recipients $2-8$ weeks after receiving the second dose of coronavirus disease 2019 (COVID19) mRNA vaccines. Among the HD and PD patients, we

Masatoshi Matsunami

matsunami.masatoshi@kameda.jp

Tomo Suzuki

suzuki.tomo@kameda.jp

Toshiki Terao

terao.toshiki@kameda.jp

Hiroshi Kuji

kuji.hiroshi@kameda.jp

Kosei Matsue

matsue.kosei@kameda.jp

1 Department of Nephrology, Kameda Medical Center, 929

Higashi-cho, Kamogawa, Chiba 296-8602, Japan

2 Department of Internal Medicine, Division of Hematology/Oncology, Kameda Medical Center, Chiba, Japan

3 Department of Urology, Kameda Medical Center, Chiba, Japan excluded a patient on treatment for immunosuppression. Due to Japan's vaccine delivery systems, group vaccination was conducted mostly with two doses of Comirnaty COVID-19 Vaccine (BioNTech-Pfizer BNT162b2).

The SARS-CoV-2-specific antibodies were evaluated and compared to that of 38 controls (a population expected to have optimal antibody response) who were volunteers that met the criteria of over 60 years of age with no evidence of kidney failure, active cancer or an ongoing treatment for immunosuppression. They were selected over a 4-month period of time by consecutive sampling from patients attending our gastroenterology outpatient clinic.

Serum samples were tested for SARS-CoV-2 antibodies using the commercially available test system Elecsys ${ }^{\circledR}$ AntiSARS-CoV-2 S RUO (Roche Diagnostics, Basel, Switzerland), measuring immunoglobulin $\mathrm{G}$ (IgG) levels against SARS-CoV-2 spike S1 subunit.

The mean age in the KTx recipients was 59 (38-81) years and lower compared to the controls, HD, and PD patients with a mean age of 74.5 (63-92), 72.9 (60-91), and 75.0 (61-95) years, respectively. Male sex was less prevalent in the controls (47.3\%) compared with the HD (65.3\%), PD $(62.9 \%)$, and KTx recipient $(57.1 \%)$ patients.

The median time on dialysis was 6.0 and 2.6 years in the HD and PD patients. In the KTx recipients, the median time after kidney transplantation was 4.0 years.

After the second vaccination, anti-SARS-CoV-2-S (Spike) IgG levels were found to be positive $(>0.8 \mathrm{U} / \mathrm{ml})$ in all 38 controls (100\%), 77 of 78 HD patients (98.7\%), and 26 of 27 PD patients (96.2\%), but only 10 of $21 \mathrm{KTx}$ recipients (47.6\%). The median S-IgG level (IQR) was 447 (IQR 308.2-1067), 200.5 (IQR 116.2-376.5), 233 (IQR 164.5-689), and 0.7 (IQR 0.4-34.7) in the control, HD, $\mathrm{PD}$, and KTx recipient groups, respectively. The median S-IgG levels were significantly lower in the HD patients $(p<0.0001)$ and KTx recipients $(p<0.0001)$ compared 


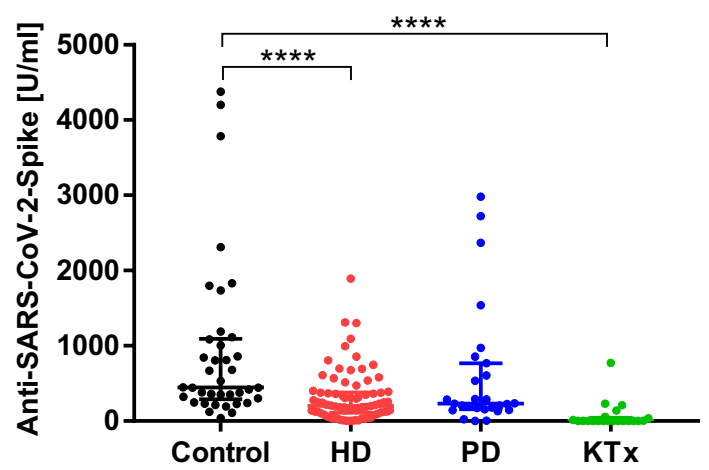

Fig. 1 Antibody response following vaccination with second dose of severe acute respiratory syndrome coronavirus 2 (SARS-CoV-2) vaccine. SARS-CoV-2 spike protein specific antibody titers were determined using Elecsys ${ }^{\circledR}$ Anti-SARS-CoV-2 S RUO. Antibody titers $>0.8 \mathrm{U} / \mathrm{ml}$ were considered as positive immune response to vaccination. Median antibody titers after second vaccination were significantly higher in the controls compared to hemodialysis patients and kidney transplant recipients. The data were analyzed using GraphPad Prism 7.0 (GraphPad Software, San Diego, CA). For comparison of the four groups (non-normally distributed samples), the data were analyzed using the non-parametric Kruskal-Wallis and post hoc Dunn's tests. ${ }^{*} p<0.05,{ }^{* *} p<0.01, * * * p<0.001, * * * * p<0.0001$ are depicted in the figure

with controls (Fig. 1). In contrast, there was no significant difference between the PD patients and controls (Fig. 1). No life-threatening allergic reaction or other side-effect was observed post-vaccination.

The KTx recipients were on a uniform immunosuppressive therapy with calcineurin inhibitor (CNI) in 20 of 21 patients, mycophenolate mofetil (MMF) or mizoribine (MZB) in 19 of 21, and steroid in 21 of 21.

Recently, studies have shown that development of antibodies due to the second dose of COVID-19 mRNA vaccines was found to be higher at $95.4 \%$ in HD patient [3]. Similarly, another study reported that antibody response in PD patients after a second dose was higher at 95.6\% [5]. In comparison, our study has shown similar results with 98.7 and $96.2 \%$ antibody positivity in HD and PD patients, respectively. Contrastingly, the antibody response to second dose COVID-19 mRNA vaccines in KTx recipients was reported to be poor in several studies, ranging from 48 to $58.8 \%$. [2, 4].

Risk factors for inadequate antibody response were older age, less time after transplant, number of used immunosuppressants, and type of immunosuppressant (antimetabolite MMF or co-stimulation blocker Belatacept) [1, 2, 4]. Although no threshold has been established for protective immunity, we found low antibody positivity (47.6\%) and titers due to second doses of the COVID-19 mRNA vaccines in KTx recipients. This may be related to the widely described impaired immune response of persistent use of immunosuppressants.
Limitation of our study includes small sample size in the KTx recipients, and types, doses, and levels of immunosuppressants were similar, which limits our conclusion on which immunosuppressant causes impaired immune response.

In conclusion, the early data that describe the antibody response after the SARS-CoV-2 vaccination suggest the response may be lower (antibody titers) in CKD patients who are receiving RRT compared to healthy patients; however, relatively high antibody positivity were observed in the dialysis patients. The response appears diminished for the KTx recipients and those on immunosuppressive therapy. For the KTx recipients and those with impaired immune responses, alternate vaccination strategies and platforms should be considered.

Acknowledgements The authors would like to thank Dr. So Nakaji for recruiting healthy volunteers from Kameda Medical Center's Gastroenterology Unit. The authors would also like to thank Drs. Mayumi Nakata, Kanako Nagaoka, Takumi Toishi, Shinnosuke Sugihara, Junko Fukuda, and Mamiko Ohara for their careful patient management at the Kameda Medical Center's Dialysis Unit.

Author contributions MM designed and wrote the manuscript. TS and $\mathrm{KM}$ contributed to the protocol design. TT and HK collected the clinical information. MM, TS, and KM analyzed the data and drafted the manuscript. All authors read and approved the final manuscript.

Funding No funding was obtained for this study.

Availability of data and materials All data supporting our findings are contained within the manuscript.

\section{Declarations}

Conflict of interest The authors have declared that no conflicts of interest exist.

Ethics approval All procedures performed in studies involving human participants were in accordance with the ethical standards of the Ethics Committee of Kameda Medical Center (Approval No. 21-025) and with the 1964 Helsinki declaration and its later amendments or comparable ethical standards.

Informed consent This ethics committee waived the requirement for written informed consent due to the retrospective nature of this study.

\section{References}

1. Stumpf J, Siepmann T, Lindner T, Karger C, Schwöbel J, Anders $\mathrm{L}$, et al. Humoral and cellular immunity to SARS-CoV-2 vaccination in renal transplant versus dialysis patients: A prospective, multicenter observational study using mRNA-1273 or BNT162b2 mRNA vaccine. Lancet Reg Health Eur. 2021;9:100178.

2. Marinaki S, Adamopoulos S, Degiannis D, Roussos S, Pavlopoulou ID, Hatzakis A, et al. Immunogenicity of SARS-CoV-2 BNT162b2 vaccine in solid organ transplant recipients. Am J Transplant. 2021;21(8):2913-5. 
3. Broseta JJ, Rodríguez-Espinosa D, Rodríguez N, Mosquera MDM, Marcos M, Egri N, et al. Humoral and cellular responses to mRNA-1273 and BNT162b2 SARS-CoV-2 vaccines administered to hemodialysis patients. Am J Kidney Dis. 2021;78(4):571-81.

4. Benotmane I, Gautier-Vargas G, Cognard N, Olagne J, Heibel F, Braun-Parvez L, et al. Low immunization rates among kidney transplant recipients who received 2 doses of the mRNA-1273 SARS-CoV-2 vaccine. Kidney Int. 2021;99(6):1498-500.

5. Agur T, Ben-Dor N, Goldman S, Lichtenberg S, Herman-Edelstein $\mathrm{M}$, Yahav D, et al. Antibody response to mRNA SARS-CoV-2 vaccine among dialysis patients-a prospective cohort study. Nephrol Dial Transplant. 2021;36(7):1347-9.

Publisher's Note Springer Nature remains neutral with regard to jurisdictional claims in published maps and institutional affiliations. 